\title{
Ganhos genéticos preditos por diferentes métodos de seleção em progênies de Eucalyptus urophylla
}

\author{
Antônio Marcos Rosado(1), Tatiana Barbosa Rosado ${ }^{(2)}$, Márcio Fernando Ribeiro Resende Júnior(2), \\ Leonardo Lopes Bhering ${ }^{(3)}$ e Cosme Damião Cruz $^{(2)}$
}

\begin{abstract}
(1)Celulose Nipo-Brasileira S.A, Rodovia BR 381, Km 172, CEP 35196-000 Belo Oriente, MG. E-mail: antonio.rosado@cenibra.com.br (2) Universidade Federal de Viçosa, Departamento de Biologia Geral, Avenida P. H. Rolfs, s/no, CEP 36571-000 Viçosa, MG. E-mail: tatianarosado@yahoo.com.br, marciopac@hotmail.com, cdcruz@ufv.br ${ }^{(3)}$ Embrapa Agroenergia, Parque Estação Biológica, s/no, CEP 70770-901 Brasília, DF. E-mail: leonardo.bhering@embrapa.br
\end{abstract}

Resumo - O objetivo deste trabalho foi avaliar parâmetros genéticos e comparar os ganhos preditos por meio de diferentes métodos de seleção em famílias de meios-irmãos de Eucalyptus urophylla. Foi utilizada seleção entre e dentro, seleção combinada e seleção com base em modelos mistos (REML/BLUP) para os caracteres diâmetro à altura do peito, altura total e volume total com casca. Foi utilizado o teste de progênie constituído de 100 famílias de meios-irmãos com 55 meses de idade, em espaçamento de $3 \times 2 \mathrm{~m}$, em delineamento de blocos ao acaso, com cinco repetições. As progênies apresentaram variabilidade genética significativa e elevada magnitude de herdabilidade para os caracteres estudados, o que evidencia alto controle genético e condições favoráveis para seleção. Todos os métodos avaliados foram eficientes para aplicação no melhoramento de eucalipto. No entanto, a seleção combinada e a seleção por modelos mistos (BLUP) proporcionam estimativas de ganhos significativamente maiores às obtidas com a seleção entre e dentro, e maior eficiência na escolha dos melhores indivíduos dentro da população.

Termos para indexação: eucalipto, ganhos de seleção, melhoramento florestal, modelos mistos.

\section{Predicted genetic gains by various selection methods in Eucalyptus urophylla progenies}

\begin{abstract}
The objective of this work was to evaluate genetic parameters and to compare predicted gains using different selection methods in half-sib families of Eucalyptus urophylla. Within and between selection, combined selection and selection based on mixed model equations (REML/BLUP) were used for the traits diameter at breast height, total height and total volume with bark. The progeny test used consisted of 100 55-month-old half-sib families distributed in a 3×2-m spacing, in randomized complete block design with five replicates. The progenies showed significant genetic variability and high heritability for the studied traits, which indicates high genetic control and favorable conditions for selection. All the methods tested were efficient in eucalyptus breeding. However, the combined selection and the selection based on mixed models (BLUP) provided gains significantly larger than those obtained with within and between selections, and were more efficient in the selection of the best individuals in the population.
\end{abstract}

Index terms: eucalyptus, genetic gains, forestry breeding, mixed models.

\section{Introdução}

Entre as espécies de eucaliptos existentes no Brasil, o Eucalyptus urophylla S.T. Blake é uma das mais plantadas devido à sua plasticidade e adaptabilidade às mais diversas regiões do país, à resistência ao cancro do eucalipto e ao potencial de utilização da madeira (Scanavaca Junior \& Garcia, 2004). No entanto, para obtenção de plantios florestais que satisfaçam às exigências do mercado, é importante considerar, além do desenvolvimento de novas técnicas de manejo, os aspectos genéticos, uma vez que a floresta é o resultado das ações e interações de seus genótipos com o ambiente (Martins et al., 2005). Nesse contexto, a seleção de genótipos superiores por melhoramento genético é uma ferramenta fundamental para gerar florestas mais produtivas e adaptadas a diferentes regiões.

A identificação de genótipos superiores requer métodos de seleção capazes de explorar eficientemente o material genético disponível, maximizando o ganho genético em relação às características de interesse (Oda et al., 2007). Diversos métodos de seleção têm

Pesq. agropec. bras., Brasília, v.44, n.12, p.1653-1659, dez. 2009 
sido empregados em eucalipto, com destaque para a seleção entre e dentro de famílias (Paula et al., 2002; Martins et al., 2003, 2005), a seleção combinada (Pires et al., 1996; Martins et al., 2001, 2005) e a seleção por modelos mistos pelo método BLUP (best linear unbiased prediction, melhor predição linear não viciada) (Garcia \& Nogueira, 2005; Rocha et al., 2006, 2007).

$\mathrm{Na}$ seleção entre e dentro de famílias, em uma primeira etapa identificam-se as melhores famílias com base na média da parcela e, posteriormente, selecionam-se, nas famílias, os indivíduos com melhor desempenho (Sampaio et al., 2000). A seleção combinada é baseada em um índice que leva em consideração, simultaneamente, o comportamento dos indivíduos e de suas famílias (Vencovsky \& Barriga, 1992). O BLUP consiste basicamente na predição de valores genéticos dos efeitos aleatórios do modelo estatístico associado às observações fenotípicas, ajustando-se os dados aos efeitos fixos e ao número desigual de informações nas parcelas por meio da metodologia de modelos mistos (Resende, 2002). A escolha do método de seleção adequado depende da magnitude e dos sentidos dos ganhos genéticos preditos e da facilidade de aplicação.

O objetivo deste trabalho foi avaliar a variabilidade genética, estimar os parâmetros genéticos e comparar os ganhos preditos para alguns caracteres de crescimento, por meio de diferentes métodos de seleção em progênies de meios-irmãos de E. urophylla.

\section{Material e Métodos}

Foram utilizadas 100 famílias de meios-irmãos de E. urophylla, cujas matrizes foram selecionadas fenotipicamente para volume de madeira. As famílias foram avaliadas em Sabinópolis, $\mathrm{MG}$, em áreas da Celulose Nipo-Brasileira S.A. (Cenibra), no teste de progênies implantado em dezembro de 2001, em delineamento de blocos ao acaso com parcelas lineares de oito plantas e cinco repetições (blocos), em espaçamento de $3 \times 2 \mathrm{~m}$.

Aos 55 meses de idade, foram avaliados $\mathrm{o}$ diâmetro à altura do peito (DAP, $\mathrm{cm}$ ); a altura total (ALT, m) e o volume individual total com casca $\left(\mathrm{VOL}, \mathrm{m}^{3}\right)$ para as 100 famílias. O volume individual total com casca foi obtido segundo a expressão: $\mathrm{VOL}=0,004761+0,000033 \times \mathrm{DAP}^{2} \times \mathrm{ALT}^{2}$.
Para testar a hipótese da existência de variância genética entre médias de famílias de meios-irmãos, foi realizada análise de variância de cada característica pelo programa Genes (Cruz, 2006) utilizando os dados de indivíduos dentro das parcelas. $\mathrm{O}$ delineamento usado foi o de blocos ao acaso segundo o modelo estatístico: $Y_{\mathrm{ijk}}=\mu+\mathrm{f}_{\mathrm{i}}+\mathrm{b}_{\mathrm{j}}+\varepsilon_{\mathrm{ij}}+\delta_{\mathrm{ijk}}$, com $\mathrm{i}=1,2, \ldots \mathrm{g}$ famílias; $\mathrm{j}=1,2, \ldots$ b blocos; e $\mathrm{k}=1,2, \ldots$ n $\mathrm{n}_{\mathrm{ij}}$ plantas por parcela. $\mathrm{Y}_{\mathrm{ijk}}$ é a observação na k-ésima planta, na i-ésima família, do j-ésimo bloco; $\mu$ é a média geral da população; $f_{i}$ é o efeito da i-ésima família, em que $f_{i} \sim$ NID $\left(0, \sigma_{\mathrm{g}}^{2}\right) ; b_{\mathrm{j}}$ é o efeito do j-ésimo bloco, em que $b_{\mathrm{j}} \sim \operatorname{NID}\left(0, \sigma_{\mathrm{b}}^{2}\right) ; \varepsilon_{\mathrm{ij}}$ é o efeito da variação ambiental entre famílias, em que $\varepsilon_{\mathrm{ij}} \sim \operatorname{NID}\left(0, \sigma_{\mathrm{e}}^{2}\right)$; e $\delta_{\mathrm{ijk}}$ é o efeito da variação entre plantas dentro de famílias, em que $\delta_{\mathrm{ijk}} \sim \operatorname{NID}\left(0, \sigma_{\mathrm{fd}}^{2}\right)$.

Os componentes de variância para cada caráter foram estimados conforme Cruz et al. (2004), em que se estimaram as variâncias de bloco, fenotípica entre médias de famílias, dentro de famílias e entre plantas no experimento, ambiental entre parcelas, genotípica entre médias de famílias, dentro de família ou entre plantas dentro de família e genética aditiva.

Os coeficientes de herdabilidade para plantas individuais dentro de famílias, no bloco, no experimento, e para médias de famílias, assim como os coeficientes de variação fenotípico, genético, ambiental e experimental, foram estimados conforme Cruz et al. (2004).

Foram estimados os ganhos de seleção, considerando as 100 famílias de meios-irmãos, em seleção de $25 \%$ entre e dentro de famílias para cada caráter, bem como para seleção com base no índice de seleção combinada e em BLUP. Todas as características foram selecionadas para obter acréscimo sobre as médias originais. $\mathrm{O}$ método de seleção entre e dentro foi utilizado conforme Cruz et al. (2004), e os ganhos de seleção foram obtidos segundo a expressão $\mathrm{GS}_{\mathrm{j}(\mathrm{i})}=\hat{\beta}_{\mathrm{j}(\mathrm{i})} \mathrm{GS}_{\mathrm{i}}$, em que $\mathrm{GS}_{\mathrm{j}(\mathrm{i})}$ é o ganho de seleção indireto no caráter j pela seleção praticada no caráter i; $\mathrm{GS}_{\mathrm{i}}$ é o ganho de seleção direto no caráter $\mathrm{i} ; \hat{\beta}_{\mathrm{j}(\mathrm{i})}=\mathrm{Côv}_{\mathrm{g}}\left(\mathrm{X}_{\mathrm{i}}, \mathrm{X}_{\mathrm{j}}\right) / \hat{\sigma}_{\mathrm{gi}}^{2}$ é o coeficiente de regressão genético, em que $C \hat{v} v_{\mathrm{g}}$ $\left(X_{i}, X_{j}\right)$ é a covariância genética (entre ou dentro) entre os caracteres i e j; e $\hat{\sigma}_{\text {gi }}^{2}$ é a variância genética (entre ou dentro) do caráter principal, sobre o qual se pratica a seleção. 
A seleção combinada para um caráter determinado consistiu no estabelecimento de um índice para cada indivíduo, com os valores que compõem o índice obtidos dos próprios indivíduos e de seus parentais.

No presente trabalho, adotou-se o índice apresentado por Pires et al. (1996), dado por $\mathrm{I}_{\mathrm{ijk}}=\mathrm{b}_{1}\left(\mathrm{Y}_{\mathrm{ijk}}-\bar{Y}_{\mathrm{j}}\right)+\mathrm{b}_{2}\left(\overline{\mathrm{Y}}_{\mathrm{i}}-\overline{\mathrm{Y}}\right)$, em que $\mathrm{I}_{\mathrm{ijk}}$ é o índice estimado do valor genético (preditor do valor genético) da k-ésima planta, da i-ésima família, no j-ésimo bloco; $Y_{\mathrm{ijk}}$ é o valor fenotípico do indivíduo ijk; $\bar{Y}_{j}$ e $Y_{i}$ são a média do bloco $\mathrm{j}$ e a média da família $i$, respectivamente; $\bar{Y}$ é a média geral da população; e $b_{1}$ e $b_{2}$ são coeficientes obtidos de modo a maximizar a correlação entre o índice (I) e o verdadeiro valor genético dos indivíduos.

Considera-se que $D_{1}=Y_{i j k}-\bar{Y}_{j}$ é o desvio do valor fenotípico individual em relação à média da repetição a que pertence, e que $D_{2}=Y_{\mathrm{ijk}}-\overline{\mathrm{Y}}_{\mathrm{i}}$ é o desvio do valor fenotípico da família em relação à média geral.

Como se deseja obter o índice de seleção combinada que possibilite melhor predição do valor genotípico individual, o agregado genotípico, pela teoria do índice, é dado por $\mathrm{H}=\mathrm{g}_{\mathrm{j} \mathrm{jk}}$, em que $\mathrm{g}_{\mathrm{ijk}}$ é o valor genotípico individual. As estimativas dos coeficientes $b_{1}$ e $b_{2}$ podem ser obtidas por meio do sistema $\mathrm{Pb}=\mathrm{Ga}$, em que

$$
\begin{aligned}
& \mathrm{b}=\left[\begin{array}{c}
\mathrm{b}_{1} \\
2 \mathrm{f}
\end{array}\right], \mathrm{P}=\left[\begin{array}{cc}
\hat{\mathrm{V}}\left(\mathrm{D}_{1}\right) & \operatorname{Côv}\left(\mathrm{D}_{1}, \mathrm{D}_{2}\right) \\
\operatorname{Côv}\left(\mathrm{D}_{1}, \mathrm{D}_{2}\right) & \hat{\mathrm{V}}\left(\mathrm{D}_{2}\right)
\end{array}\right] \\
& \mathrm{G}=\left[\begin{array}{c}
\operatorname{Cov}_{\mathrm{g}}\left(\mathrm{D}_{1}, \mathrm{~g}_{\mathrm{ijk}}\right) \\
\operatorname{Cov}_{\mathrm{g}}\left(\mathrm{D}_{2}, \mathrm{~g}_{\mathrm{ijk}}\right)
\end{array}\right] \text { e } \mathrm{a}=[1] .
\end{aligned}
$$

O ganho de seleção combinado (com base no índice) foi estimado por meio de GS $=\mathrm{DS}_{\mathrm{I}}$, em que $\mathrm{DS}_{\mathrm{I}}$ é o diferencial de seleção com base no índice combinado, ou seja, $\mathrm{DS}_{\mathrm{I}}=\overline{\mathrm{I}}_{\mathrm{s}}-\overline{\mathrm{I}}$, em que $\overline{\mathrm{I}}_{\mathrm{s}}$ é o valor médio do índice considerando-se apenas os indivíduos selecionados, e $\overline{\mathrm{I}}$ é o valor médio do índice (igual a zero para os índices considerados na análise).

Para a estimativa das variâncias e covariâncias, as quais são requeridas para predição dos valores genéticos pela metodologia de modelos mistos, utilizou-se o software SELEGEM, conforme Resende \& Fernandes (1999), realizando as análises pelo método da máxima verossimilhança restrita ("restricted maximum likelihood", REML) (Patterson \& Thompson, 1971).

Para o teste de progênie no delineamento de blocos ao acaso com várias plantas por parcela, e considerando a seleção por valores genéticos aditivos para famílias de meios-irmãos, todas as análises foram feitas usando o SELEGEM e o modelo estatístico (modelo linear misto) segundo Resende \& Fernandes (1999), que equivale a $\mathrm{y}=\mathrm{Xb}+\mathrm{Za}+\mathrm{Wp}+\mathrm{e}$, em que yé o vetor de dados; b é o vetor de efeitos fixos (média geral e efeitos de bloco); a é o vetor de valores genéticos aditivos (aleatório); $p$ é o vetor de efeitos de famílias (aleatórios); e é o vetor de erros aleatórios; e X, Z e W são matrizes de incidência para $b$, a e p, respectivamente.

As equações de modelo misto para predição dos valores genéticos pelo procedimento de BLUP sob modelo individual são:

$\left[\begin{array}{c}\hat{b} \\ \hat{a} \\ \hat{p}\end{array}\right]=\left[\begin{array}{ccc}X^{\prime} X & X^{\prime} Z & X^{\prime} W \\ Z^{\prime} X & Z^{\prime} Z+A^{-1} \lambda_{1} & Z^{\prime} W \\ W^{\prime} X & W^{\prime} Z & W^{\prime} W+I \lambda_{2}\end{array}\right]^{-1} \cdot\left[\begin{array}{c}X^{\prime} y \\ Z^{\prime} y \\ W^{\prime} y\end{array}\right]$ $\lambda_{1}=\sigma_{\mathrm{ec}}^{2} / \sigma_{\mathrm{p}}^{2}=\left[1-\mathrm{h}^{2}-\mathrm{p}^{2}\right] / \mathrm{h}^{2} ; \lambda_{2}=\sigma_{\mathrm{ec}}^{2} / \sigma_{\mathrm{p}}^{2}=\left[1-\mathrm{h}^{2}-\mathrm{p}^{2}\right] / \mathrm{p}^{2} ;$ $h^{2}=\hat{\sigma}_{a}^{2} /\left[\hat{\sigma}_{a}+\hat{\sigma}_{p}^{2}+\hat{\sigma}_{\mathrm{e}}^{2}\right]$ é a herdabilidade individual no sentido restrito no bloco; $p^{2}=\hat{\sigma}_{p}^{2} /\left[\hat{\sigma}_{a}+\hat{\sigma}_{p}^{2}+\hat{\sigma}_{c}^{2}\right]$ é a correlação devida ao ambiente comum da parcela, em que $\sigma_{\mathrm{a}}^{2}$ é a variância genética aditiva; $\sigma_{\mathrm{p}}^{2}$ é a variância entre parcelas; $\sigma_{\mathrm{ee}}^{2}$ é a variância ambiental entre famílias; $\sigma_{\mathrm{e}}^{2}$ é a variância residual (ambiental dentro de parcelas + não aditiva); e A é a matriz de parentesco genético aditivo entre os indivíduos.

Segundo Resende (2000), as soluções para as equações de modelo misto devem ser obtidas por métodos iterativos de resolução de sistemas de equações lineares, da seguinte forma:

$\sigma_{\mathrm{e}}^{2}=\left[\mathrm{y}^{\prime} \mathrm{y}-\hat{\mathrm{b}}^{\prime} \mathrm{X}^{\prime} \mathrm{y}-\hat{a}^{\prime} Z^{\prime} \mathrm{y}-\hat{c}^{\prime} \mathrm{W}^{\prime} \mathrm{y}\right] /[\mathrm{N}-\mathrm{r}(\mathrm{x})]$;

$\sigma_{\mathrm{p}}^{2}=\left[\hat{\mathrm{c}}^{\prime} \hat{\mathrm{c}}+\tilde{\sigma}_{\mathrm{e}}^{2} \operatorname{tr} \mathrm{C}^{33}\right] / \mathrm{s}$;

$\sigma_{\mathrm{a}}^{2}=\left[\hat{a}^{\prime} \mathrm{A}^{-1} \hat{\mathrm{a}}+\hat{\sigma}_{\mathrm{e}}^{2} \operatorname{tr}\left(\mathrm{A}^{-1} \mathrm{C}^{22}\right)\right] / \mathrm{q}$ é a variância ambiental entre famílias; $\sigma_{\mathrm{e}}^{2}$ é a variância residual (ambiental dentro de parcelas + não aditiva), $\mathrm{C}^{22}$ e $\mathrm{C}^{33}$ advêm de:

$\mathrm{C}^{-1}=\left[\begin{array}{lll}\mathrm{C}_{11} & \mathrm{C}_{12} & \mathrm{C}_{13} \\ \mathrm{C}_{21} & \mathrm{C}_{22} & \mathrm{C}_{23} \\ \mathrm{C}_{31} & \mathrm{C}_{32} & \mathrm{C}_{33}\end{array}\right]^{-1}=\left[\begin{array}{lll}\mathrm{C}^{11} & \mathrm{C}^{12} & \mathrm{C}^{13} \\ \mathrm{C}^{21} & \mathrm{C}^{22} & \mathrm{C}^{23} \\ \mathrm{C}^{31} & \mathrm{C}^{32} & \mathrm{C}^{33}\end{array}\right]$

C é a matriz dos coeficientes das equações de modelo misto; tr é o operador traço matricial; $\mathrm{r}(\mathrm{x})$ é o posto da matriz X; N é o número total de dados; q é o número de indivíduos; s é o número de parcelas.

\section{Resultados e Discussão}

A análise de variância indicou a existência de variância genética significativa a 5\% de probabilidade 
para os três caracteres avaliados, indicando a possibilidade de obter ganhos com a seleção (Tabela 1). Os valores do coeficiente de variação experimental $\left(\mathrm{CV}_{\mathrm{ex}}\right)$ obtidos para diâmetro à altura do peito, altura total e volume individual total com casca podem ser considerados médios, conforme os valores normalmente encontrados para o gênero Eucalyptus (Garcia \& Nogueira, 2005), o que evidencia precisão na obtenção e análise dos dados e atribui confiabilidade aos resultados. As médias dos caracteres também estão entre os valores encontrados por outros autores para a espécie na idade avaliada (Pires et al., 1996; Resende \& Fernandes, 1999; Rocha et al., 2007).

As estimativas das variâncias (Tabela 2) evidenciaram que os três caracteres em estudo apresentaram taxas de controle genético similares, guardadas as devidas escalas, por serem caracteres correlacionados. Isso indica que, em se tratando de seleção para crescimento, há boas chances de sucesso nos três caracteres pela seleção de apenas um. As estimativas das variâncias genéticas aditivas foram similares para as características diâmetro à altura do peito e altura total, bem como as variâncias fenotípicas entre famílias, o que evidencia a possibilidade de uso de ambos na predição de valores genéticos dos indivíduos candidatos à seleção. Como as variâncias fenotípica total em virtude do efeito de bloco e ambiental entre parcelas tenderam a zero, pode-se concluir que há baixa magnitude para o efeito de blocos e boa eficiência em termos de precisão experimental.

$\mathrm{O}$ coeficiente de variação genético $\left(\mathrm{CV}_{\mathrm{g}}\right)$ é outro parâmetro comumente usado para comparar a

Tabela 1. Análise de variância para diâmetro à altura do peito (DAP, cm), altura total (ALT, m) e volume total com casca $\left(\mathrm{VOL}, \mathrm{m}^{3}\right)$ em famílias de meios-irmãos de Eucalyptus urophylla ${ }^{(1)}$.

\begin{tabular}{lcccc}
\hline Fonte de variação & \multirow{2}{*}{ GL } & \multicolumn{3}{c}{ Quadrados médios } \\
\cline { 3 - 5 } & & DAP & ALT & VOL \\
\hline Blocos & 4 & 17,29 & 17,13 & $4,7 \times 10^{-3}$ \\
Família & 99 & $21,37 *$ & $23,57 *$ & $6,7 \times 10^{-3} *$ \\
Entre família & 396 & 8,63 & 10,01 & $2,5 \times 10^{-3}$ \\
Dentro de família & 2910 & 7,74 & 5,25 & $1,9 \times 10^{-3}$ \\
\hline Média & & 12,10 & 16,63 & $9,4 \times 10^{-2}$ \\
$\mathrm{CV}_{\mathrm{ex}}(\%)$ & 9,51 & 7,45 & 20,79 \\
${ }^{(1)} \mathrm{CV}_{\text {ex }}$, coeficiente de variação & \multirow{2}{*}{ probabilidade. } & &
\end{tabular}

Pesq. agropec. bras., Brasília, v.44, n.12, p.1653-1659, dez. 2009 variabilidade genética expressa para cada caráter, conforme destacado por Resende (2000). Ademais, segundo Vencovsky (1987), quando a relação entre $\mathrm{CV}_{\mathrm{g}}$ e o coeficiente de variação ambiental $\left(\mathrm{CV}_{\mathrm{e}}\right)$ tende a 1 ou mais, há uma situação favorável para a obtenção de ganhos na seleção. Tomando a relação entre $\mathrm{CV}_{\mathrm{g}}$ e $\mathrm{CV}_{\mathrm{e}}$ para todos os caracteres, com exceção do coeficiente de variação genética entre famílias $\left(\mathrm{CV}_{\text {ge }}\right)$ da altura total, tanto o $\mathrm{CV}_{\text {ge }}$ quanto o coeficiente de variação genética dentro de famílias $\left(\mathrm{CV}_{\mathrm{gd}}\right)$ foram superiores ao $\mathrm{CV}_{\mathrm{e}}$ (Tabela 3 ), o que indica ganhos favoráveis com a seleção. Ademais, com o fato de todos os caracteres apresentarem o $\mathrm{CV}_{\mathrm{gd}}$ superior ao $\mathrm{CV}_{\mathrm{ge}}$, espera-se que a seleção entre e dentro de famílias promova maiores progressos com a seleção do que somente a seleção entre famílias.

As estimativas de herdabilidade, tomadas em conjunto, foram elevadas, o que evidencia um alto controle genético e permite predizer condições favoráveis para a seleção para todos os caracteres em estudo (Tabela 4). As estimativas de herdabilidade com base nas médias de famílias foram, em geral, superiores àquelas dentro de famílias, o que corrobora outros estudos com diferentes espécies, como em Eucalyptus grandis (Martins et al., 2001, 2005) e E. camaldulensis (Paula et al., 2002), o que indica que a seleção com base nas médias das famílias deve ser mais eficiente que dentro de famílias, considerando uma mesma intensidade de seleção. Nesse caso, pode-se combinar a seleção entre e dentro, para

Tabela 2. Estimativas das variâncias para diâmetro à altura do peito (DAP, cm), altura total (ALT, m) e volume total com casca (VOL, $\mathrm{m}^{3}$ ) em famílias de meios-irmãos de Eucalyptus urophylla.

\begin{tabular}{lccc}
\hline Variância $^{(1)}$ & DAP & ALT & VOL \\
\hline$\hat{\sigma}_{\mathrm{gm}}^{2}$ & 0,39 & 0,42 & $1,0 \times 10^{-4}$ \\
$\hat{\sigma}_{\mathrm{gd}}^{2}$ & 7,61 & 4,63 & $1,9 \times 10^{-3}$ \\
$\hat{\sigma}_{\mathrm{a}}^{2}$ & 1,56 & 1,66 & $5,0 \times 10^{-4}$ \\
$\hat{\sigma}_{\mathrm{fm}}^{2}$ & 0,54 & 1,14 & $2,0 \times 10^{-4}$ \\
$\hat{\sigma}_{\mathrm{fd}}^{2}$ & 7,74 & 5,35 & $2,0 \times 10^{-3}$ \\
$\hat{\sigma}_{\mathrm{ft}}^{2}$ & 8,28 & 6,49 & $2,2 \times 10^{-3}$ \\
$\hat{\sigma}_{\mathrm{b}}^{2}$ & 0,01 & 0,01 & 0 \\
$\hat{\sigma}_{\mathrm{ee}}^{2}$ & 0,14 & 0,71 & 0 \\
${ }^{(1)} \hat{\sigma}_{\text {gmm }}^{2}$, variância genotípica entre médias & de famílias; $\hat{\sigma}_{\mathrm{gd}}^{2}$, variância \\
genotípica dentro de família; $\hat{\sigma}_{\mathrm{a}}^{2}$, variância genética aditiva; $\hat{\sigma}_{\mathrm{fm}}^{2}$, variância \\
fenotípica entre médias de famílias; $\hat{\sigma}_{\mathrm{fd}}^{2}$, variância fenotípica dentro de \\
famílias; $\hat{\sigma}_{\mathrm{ft}}^{2}$, variância fenotípica total; $\hat{\sigma}_{\mathrm{b}}^{2}$, variância fenotípica em virtude \\
do efeito de bloco e $\hat{\sigma}_{\mathrm{ee}}^{2}$ variância ambiental entre parcelas.
\end{tabular}


explorar adequadamente a variabilidade, elevando o ganho genético total.

$\mathrm{Na}$ comparação dos ganhos totais com todos os critérios de seleção estudados (Tabela 5), verificaram-se ganhos positivos para todas as características, com os maiores ganhos obtidos com a seleção combinada e pelo método BLUP, para todos os caracteres. Foram selecionadas 50 plantas, que corresponderam a $6,25 \%$ da população. Os maiores ganhos, tanto para seleção entre e dentro como para seleção combinada, foram para volume individual total com casca, resultado compatível com a alta variação genética desta característica.

A seleção combinada proporcionou estimativas de ganhos superiores aos da seleção entre e dentro para todos os caracteres, o que está de acordo com outros estudos (Sampaio et al., 2000; Martins et al., 2001). Martins et al. (2001), ao estudar progênies de E. grandis, verificaram que o processo de seleção

Tabela 3. Estimativas dos coeficientes de variação para diâmetro à altura do peito $(\mathrm{DAP}, \mathrm{cm})$, altura total (ALT, m) e volume total com casca (VOL, $\mathrm{m}^{3}$ ) em famílias de meios-irmãos de Eucalyptus urophylla.

\begin{tabular}{lrrr}
\hline $\mathrm{CV}(\%)^{(1)}$ & $\mathrm{DAP}$ & $\mathrm{ALT}$ & $\mathrm{VOL}$ \\
\hline $\mathrm{CV}_{\mathrm{ge}}$ & 5,16 & 3,87 & 12,11 \\
$\mathrm{CV}_{\mathrm{gd}}$ & 22,79 & 12,94 & 45,91 \\
$\mathrm{CV}_{\mathrm{fd}}$ & 22,99 & 13,91 & 46,93 \\
$\mathrm{CV}_{\mathrm{fex}}$ & 23,79 & 15,32 & 49,42 \\
$\mathrm{CV}_{\mathrm{e}}$ & 3,05 & 5,08 & 9,72 \\
$\mathrm{CV}_{\mathrm{ge}} / \mathrm{CV}_{\mathrm{e}}$ & 1,69 & 0,76 & 1,25 \\
$\mathrm{CV}_{\mathrm{gd}} / \mathrm{CV}_{\mathrm{e}}$ & 7,46 & 2,54 & 4,73 \\
\hline
\end{tabular}

${ }^{(1)} \mathrm{CV}$, coeficiente de variação; $\mathrm{CV}_{\mathrm{ge}}$, genético entre famílias; $\mathrm{CV}_{\mathrm{gd}}$, genético dentro de família, $\mathrm{CV}_{\mathrm{fd}}$, fenotípico entre plantas dentro de família; $\mathrm{CV}_{\mathrm{fex}}$, fenotípico entre plantas dentro do experimento; $\mathrm{CV}_{\mathrm{e}}$, ambiental; $\mathrm{CV}_{\mathrm{ge}}$ / $\mathrm{CV}_{\mathrm{e}}$ e $\mathrm{CV}_{\mathrm{gd}} / \mathrm{CV}_{\mathrm{e}}$, relação entre os coeficientes de variação genético entre e dentro de famílias e ambiental.

Tabela 4. Estimativas dos coeficientes de herdabilidade para diâmetro à altura do peito (DAP, $\mathrm{cm}$ ), altura total (ALT, m) e volume total com casca $\left(\mathrm{VOL}, \mathrm{m}^{3}\right)$ em famílias de meios-irmãos de Eucalyptus urophylla.

\begin{tabular}{lccc}
\hline Coeficiente de herdabilidade $^{(1)}$ & DAP & ALT & VOL \\
\hline $\mathrm{h}_{\mathrm{m}}^{2}$ & 0,59 & 0,58 & 0,63 \\
$\mathrm{~h}_{\mathrm{d}}^{2}$ & 0,15 & 0,23 & 0,20 \\
$\mathrm{~h}_{\mathrm{ex}}^{2}$ & 0,19 & 0,26 & 0,24 \\
$\mathrm{~h}_{\mathrm{b}}^{2}$ & 0,19 & 0,26 & 0,24 \\
\hline
\end{tabular}

${ }^{(1)} h_{m}^{2}$, coeficiente de herdabilidade de médias de famílias; $h_{d}^{2}$, coeficiente de herdabilidade dentro de famílias; $h_{\mathrm{ex}}^{2}$, coeficiente de herdabilidade de plantas no experimento; $h_{b}^{2}$ coeficiente de herdabilidade de plantas no bloco. combinada é especialmente recomendado quando se tem baixa variabilidade dentro de famílias.

A maior eficiência da seleção combinada é devida ao índice no qual ela se baseia, que leva em consideração, simultaneamente, medidas genéticas (valores genéticos líquidos) do comportamento dos indivíduos e de suas famílias, e não medidas fenotípicas, as quais estão sujeitas a maior influência dos efeitos ambientais, como na seleção entre e dentro (Vencovsky \& Barriga, 1992).

A seleção com base no BLUP proporcionou estimativas de ganho bem próximas às da seleção combinada (Tabela 5), o que evidencia que ambos são eficientes no melhoramento de Eucalyptus.

Neste trabalho, como o ensaio foi balanceado e ortogonal, a ordem de classificação dos genótipos não se alterou em relação aos métodos de seleção. No entanto, a ocorrência de desbalanceamento não planejado, decorrente da perda de parcelas, é um fato normal nos experimentos com plantas perenes. Ademais, nas fases preliminares do processo seletivo, quando os genótipos são numerosos e ainda têm natureza aleatória (Piepho, 1994), é comum o uso de delineamentos não ortogonais em blocos incompletos balanceados e em blocos incompletos parcialmente balanceados. Também têm ganhado aplicação crescente os delineamentos aumentados, os quais, por construção, são desbalanceados e não ortogonais. Nesses casos, a possibilidade de classificações genotípicas diferenciadas entre as

Tabela 5. Estimativas das médias dos indivíduos selecionados (MS), dos ganhos esperados (GS) e percentagem das estimativas dos ganhos esperados (GS, \%) pela seleção entre e dentro, combinada e pelo BLUP, para diâmetro à altura do peito (DAP, cm), altura total (ALT, m) e volume total com casca $\left(\mathrm{VOL}, \mathrm{m}^{3}\right)$, em famílias de meios-irmãos de Eucalyptus urophylla.

\begin{tabular}{lrcr}
\hline Método de seleção & DAP & ALT & \multicolumn{1}{c}{ VOL } \\
\hline \multicolumn{4}{c}{ Seleção entre e dentro } \\
MS & 13,15 & 17,69 & 0,112 \\
GS & 1,03 & 1,00 & 0,020 \\
GS (\%) & 8,50 & 6,04 & 23,680 \\
\hline \multicolumn{4}{c}{ Seleção combinada } \\
MS & 16,02 & 19,93 & 0,180 \\
GS & 1,34 & 1,31 & 0,033 \\
GS (\%) & 11,11 & 7,88 & 29,920 \\
\hline & \multicolumn{4}{c}{} \\
MS & 15,97 & 19,90 & 0,175 \\
GS & 1,32 & 1,30 & 0,027 \\
GS (\%) & 10,93 & 7,81 & 31,700 \\
\hline
\end{tabular}


duas abordagens analíticas é uma realidade, e os ganhos preditos com a metodologia de modelos mistos é sempre superior (Garcia \& Nogueira, 2005). Essa metodologia tem-se tornado mais acessível aos usuários graças à sua implementação em sistemas estatístico-computacionais e tem sido utilizada para a seleção em diversas culturas perenes, como pinheiro (Missio et al., 2004), eucalipto (Garcia \& Nogueira, 2005; Rocha et al., 2006, 2007), pupunha (Farias Neto \& Resende, 2001) e café (Petek et al., 2008).

\section{Conclusões}

1. As famílias de meios-irmãos de Eucalyptus urophylla apresentam variabilidade genética para diâmetro à altura do peito, altura total e volume total com casca.

2. As estimativas de herdabilidades para os caracteres estudados com base nas médias de famílias de meios-irmãos de E. urophylla evidenciam alto controle genético e condições favoráveis para seleção.

3. A seleção sobre o volume total com casca possibilita maiores estimativas de ganhos genéticos em relação à altura e ao diâmetro à altura do peito.

4. A seleção combinada e a seleção por modelos mistos (BLUP) proporcionam estimativas de ganhos significativamente maiores que as obtidas com a seleção entre e dentro e maior eficiência na escolha dos melhores indivíduos dentro da população.

\section{Agradecimentos}

Ao Conselho Nacional de Desenvolvimento Científico e Tecnológico, pelo apoio financeiro.

\section{Referências}

CRUZ, C.D. Programa GENES: biometria. Viçosa: UFV, 2006. 382p.

CRUZ, C.D.; REGAZZI, A.J.; CARNEIRO, P.C.S. Modelos biométricos aplicados ao melhoramento genético. 3.ed. Viçosa: UFV, 2004. 480p.

FARIAS NETO, J.T. de; RESENDE, M.D.V. de. Aplicação da metodologia de modelos mistos (REML/BLUP) na estimação de componentes de variância e predição de valores genéticos em pupunheira (Bactris gasipaes). Revista Brasileira de Fruticultura, v.23, p.320-324, 2001.
GARCIA, C.H.; NOGUEIRA, M.C.S. Utilização da metodologia REML/BLUP na seleção de clones de eucalipto. Scientia Forestalis, v.68, p.107-112, 2005.

MARTINS, I.S.; CRUZ, C.D.; REGAZZI, A.J.; PIRES, I.E. Eficiência da seleção univariada direta e indireta e de índices de seleção em Eucalyptus grandis. Revista Árvore, v.27, p.327-333, 2003.

MARTINS, I.S.; CRUZ, C.D.; ROCHA, M. das G. de B.; REGAZZI, A.J.; PIRES, I.E. Comparação entre os processos de seleção entre e dentro e o de seleção combinada, em progênies de Eucalyptus grandis. Cerne, v.11, p.16-24, 2005.

MARTINS, I.S.; MARTINS, R. de C.C.; CORREIA, H. de S. Comparação entre seleção combinada e seleção direta em Eucalyptus grandis, sob diferentes intensidades de seleção. Floresta e Ambiente, v. 8, p.36-43, 2001.

MISSIO, R.F.; DIAS, L.A. dos S.; MORAES, M.L.T. de; RESENDE, M.D.V. de. Selection of Pinus caribaea var. bahamensis progenies based on the predicted genetic value. Crop Breeding and Applied Biotechnology, v.4, p.399-407, 2004.

ODA, S.; MELLO, E.J.; SILVA, J.F.; SOUZA, I.C.G. Melhoramento florestal. In: BORÉM, A. (Ed.). Biotecnologia Florestal. Viçosa: UFV, 2007. p.51-71.

PATTERSON, H.D.; THOMPSON, R. Recovery of inter-block information when block sizes are unequal. Biometrika, v.58, p.545-554, 1971.

PAULA, R.C. de; PIRES, I.E.; BORGES, R. de C.G.; CRUZ, C.D. Predição de ganhos genéticos em melhoramento florestal. Pesquisa Agropecuária Brasileira, v.37, p.159-165, 2002.

PETEK, M.R.; SERA, T.; FONSECA, I.C. de B. Predição de valores genéticos aditivos na seleção visando obter cultivares de café mais resistentes à ferrugem. Bragantia, v.67, p.133-140, 2008.

PIEPHO, H.P. Best linear unbiased prediction (BLUP) for regional yield trials: a comparison to additive main effects multiplicative interaction (AMMI) analysis. Theoretical and Applied Genetics, v.89, p.647-654, 1994.

PIRES, I.E.; CRUZ, C.D.; BORGES, R.C.G.; REGAZZI, A.J. Índice de seleção combinada aplicado ao melhoramento genético de Eucalyptus spp. Revista Árvore, v.20, p.191-197, 1996.

RESENDE, M.D.V. de. Análise estatística de modelos mistos via REML/BLUP na exper imentação em melhoramento de plantas perenes. Colombo: Embrapa Florestas, 2000. 101p. (Embrapa Florestas. Documentos, 47).

RESENDE, M.D.V. de. Genética biométrica e estatística no melhoramento de plantas perenes. Brasília: Embrapa Informação Tecnológica; Colombo: Embrapa Florestas, 2002. 975p.

RESENDE, M.D.V. de; FERNANDES, J.S.C. Procedimento BLUP individual para delineamentos experimentais aplicados ao melhoramento florestal. Revista de Matemática e Estatística, v.17, p.89-107, 1999. 
ROCHA, M. das G. de B.; PIRES, I.E.; ROCHA, R.B.; XAVIER, A.; CRUZ, C.D. Avaliação genética de progênies de meio-irmãos de Eucalyptus grandis por meio dos procedimentos REML/BLUP e da ANOVA. Scientia Forestalis, v.71, p.99-107, 2006.

ROCHA, M. das G. de B.; PIRES, I.E.; ROCHA, R.B.; XAVIER, A.; CRUZ, C.D. Seleção de genitores de Eucalyptus grandis e de Eucalyptus urophylla para a produção de híbridos interespecíficos utilizando REML/BLUP e informação de divergência genética. Revista Árvore, v.31, p.977-987, 2007.

SAMPAIO, P. de T.B.; RESENDE, M.D.V. de; ARAÚJO, A.J. de. Estimativas de parâmetros genéticos e métodos de seleção para o melhoramento genético de Pinus caribaea var. hondurensis. Pesquisa Agropecuária Brasileira, v.35, p.2243-2253, 2000.

SCANAVACA JUNIOR, L.; GARCIA, J.N. Determinação das propriedades físicas emecânicas damadeira de Eucalyptusurophylla. Scientia Forestalis, v.65, p.120-129, 2004.

VENCOVSKY, R. Herança quantitativa. In: PATERNIANI, E. (Coord.). Melhoramento e a produção de milho no Brasil. Piracicaba: Fundação Cargill, 1987. p.137-214.

VENCOVSKY, R.; BARRIGA, P. Genética biométrica no fitomelhoramento. Ribeirão Preto: Sociedade Brasileira de Genética, 1992. 486p.

$\overline{\text { Recebido em } 25 \text { de março de } 2009 \text { e aprovado em } 21 \text { de novembro de } 2009}$ 